\title{
Irreversible reorganization in a supercooled liquid originates from localized soft modes
}

\author{
ASAPH WIDMER-COOPER ${ }^{1}$, HEIDI PERRY2 ${ }^{2}$ PETER HARROWELL ${ }^{1 *}$ AND DAVID R. REICHMAN2* \\ ${ }^{1}$ School of Chemistry, University of Sydney, Sydney, New South Wales 2006, Australia \\ ${ }^{2}$ Department of Chemistry, Columbia University, 3000 Broadway, New York 10027, USA \\ *e-mail: peter@chem.usyd.edu.au; reichman@chem.columbia.edu
}

The transition of a fluid to a rigid glass on cooling is a common route of transformation from liquid to solid that embodies the most poorly understood features of both phases ${ }^{1-3}$. From the liquid perspective, the puzzle is to understand stress relaxation in the disordered state. From the perspective of solids, the challenge is to extend our description of structure and its mechanical consequences to materials without long-range order. Using computer simulations, we show that the localized low-frequency normal modes of a configuration in a supercooled liquid are causally correlated to the irreversible structural reorganization of the particles within this configuration. We also demonstrate that the spatial distribution of these soft local modes can persist in spite of significant particle reorganization. The consequence of these two results is that it is now feasible to construct a theory of relaxation length scales in glass-forming liquids without recourse to dynamics and to explicitly relate molecular properties to their collective relaxation.

A crucial concept ${ }^{4-9}$ for the transition between liquid and glass is that the dramatic increase in viscosity of a supercooled liquid as it approaches vitrification is caused by the growth of localized domains of particles that must rearrange for the liquid to flow. Locating a causal link between local structure and such dynamical heterogeneities has proven elusive ${ }^{10,11}$. Recently, a fruitful computational approach to isolating the structural origin of dynamical heterogeneity has been put forward in the dual notions of an 'iso-configurational ensemble' and 'propensity for motion' ${ }^{\prime 2}$. The iso-configurational ensemble refers to the ensemble of trajectories that are run from an identical configuration of particles with random initial momenta sampled from the equilibrium Boltzmann distribution. Propensity refers to the mean squared displacement of individual particles when averaged over the ensemble at a given time scale. The heterogeneous character and increased clustering showed in the spatial propensity maps has established that the spatial distribution of these dynamic heterogeneities can be explicitly attributed to structural features, as yet unidentified.

Our goal is to understand that aspect of dynamic heterogeneity directly associated with structural relaxation. To this end we are interested in motions that (1) involve local reorganization of particle configurations (here measured in terms of changes in nearest-neighbour pairings ${ }^{13}$ ) and (2) are irreversible over some observation time ${ }^{14}$ and, hence, contribute to relaxation. To investigate this we consider a two-dimensional binary mixture of soft discs whose transition from liquid to glass-like behaviour has been well characterized ${ }^{15}$. The temperature units and the timescale $\tau$ are defined in the Methods summary. We first establish how many nearest neighbours must be lost around a given particle before the probability of the tagged particle recovering its original environment falls below 5\%. For the binary mixture of soft discs under investigation here, this threshold is four neighbours (see the Supplementary Information). Equipped with this measure, we can determine how the irreversible reorganization (IR) is distributed in a given configuration. We record, over an ensemble of 100 iso-configurational runs, the number of runs in which each particle meets the irreversibility criterion within a time interval of $200 \tau$ (this corresponds to the time at which the peak of the non-Gaussian parameter ${ }^{9}$ occurs at this temperature and represents a timescale of about 2,000 collision events). Maps of the $\log$ of this probability distribution for six configurations at a reduced temperature $T=0.4$ are shown in Fig. 1 . The IRs mapped in Fig. 1 are elementary components of the slow structural relaxation characterized by a time $\tau_{\alpha}(=673 \tau$ for the simulations reported here ${ }^{15}$ ).

What aspect of the initial configuration is responsible for the observed spatial distribution of irreversible events? Previously, we have demonstrated that the spatial distribution of the free volume $^{11}$ and local potential energy ${ }^{10}$ do not show any significant correlation with the spatial distribution of the propensity. To move beyond these purely local measures, we have determined the normal modes for the local potential energy minimum (the 'inherent structure') associated with a number of initial configurations. We shall refer to these as quenched normal modes. The participation fraction of particle $i$ in eigenmode $\mathbf{e}_{\omega}$ is given by $p_{i}=\left|\mathbf{e}_{\omega}^{i}\right|^{2}$. In Fig. 2 we have plotted maps of the particle participation fractions summed over the 30 lowest-frequency modes for each of our initial configurations. An examination of the individual mode eigenfunctions (see the Supplementary Information) indicates that these low-frequency modes include both localized and delocalized character ${ }^{16-18}$.

To establish the connection between the mode structure and the subsequent IR, we have plotted in Fig. 3 the positions (white circles) of those particles with $\geq 0.01$ probability of meeting our IR condition during the entire $200 \tau$ interval in the iso-configurational ensemble on top of the maps of the participation fractions for the low-frequency modes at time $t=0$. Note that the majority contribution to the IR map comes from particles that lost their fourth neighbour late in the trajectory. While these results do not address the question of when or even whether a given soft local mode will become involved in IR, they do strongly support a picture in which the IR of a configuration originates from these modes. 

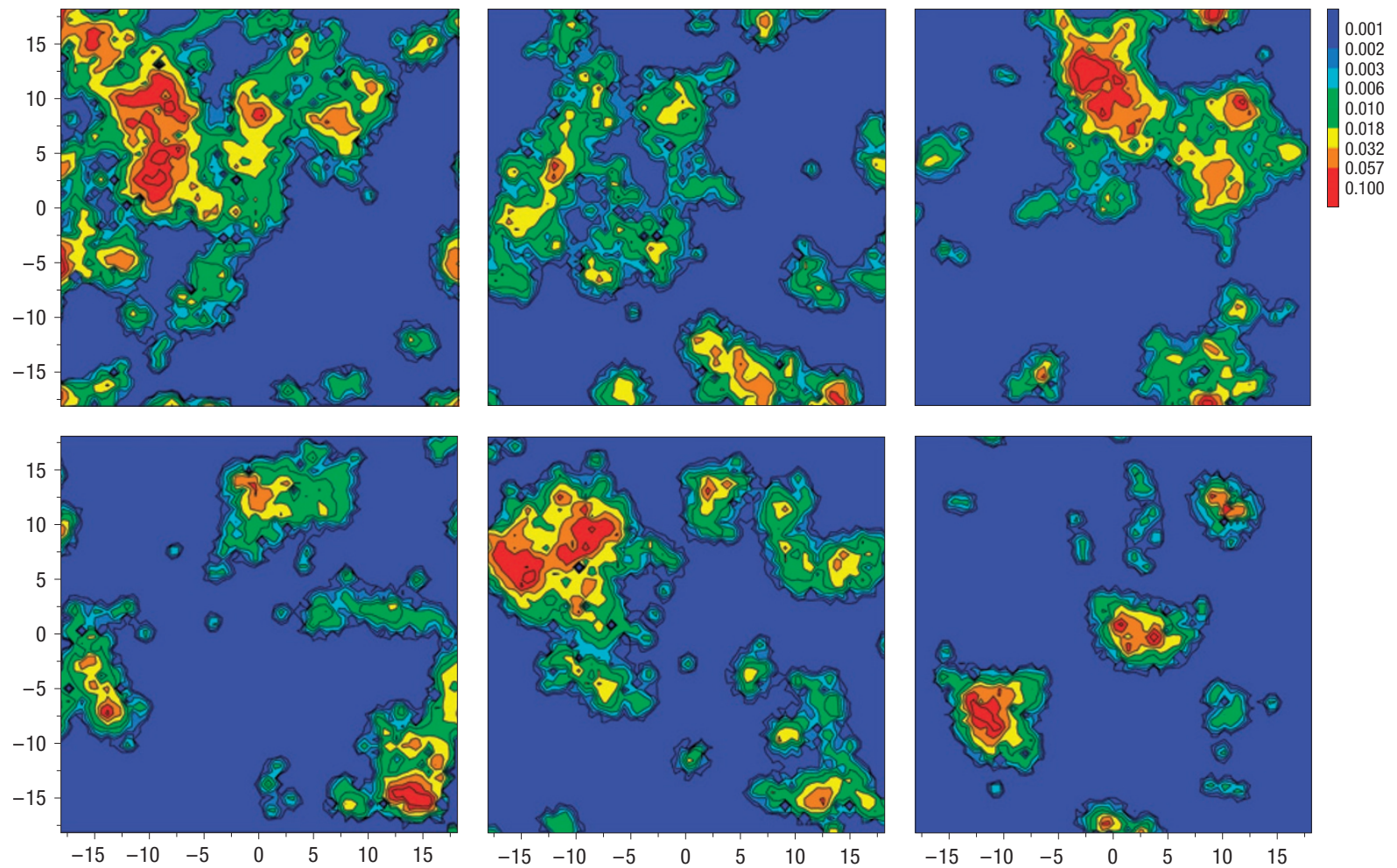

Figure 1 The spatial distribution of irreversible reorganization (IR). Contour plots of the probability $\left(\log _{10}\right)$ of a particle losing four original neighbours, the criterion for IR, within $200 \tau$ over 100 iso-configurational runs for six different initial configurations.
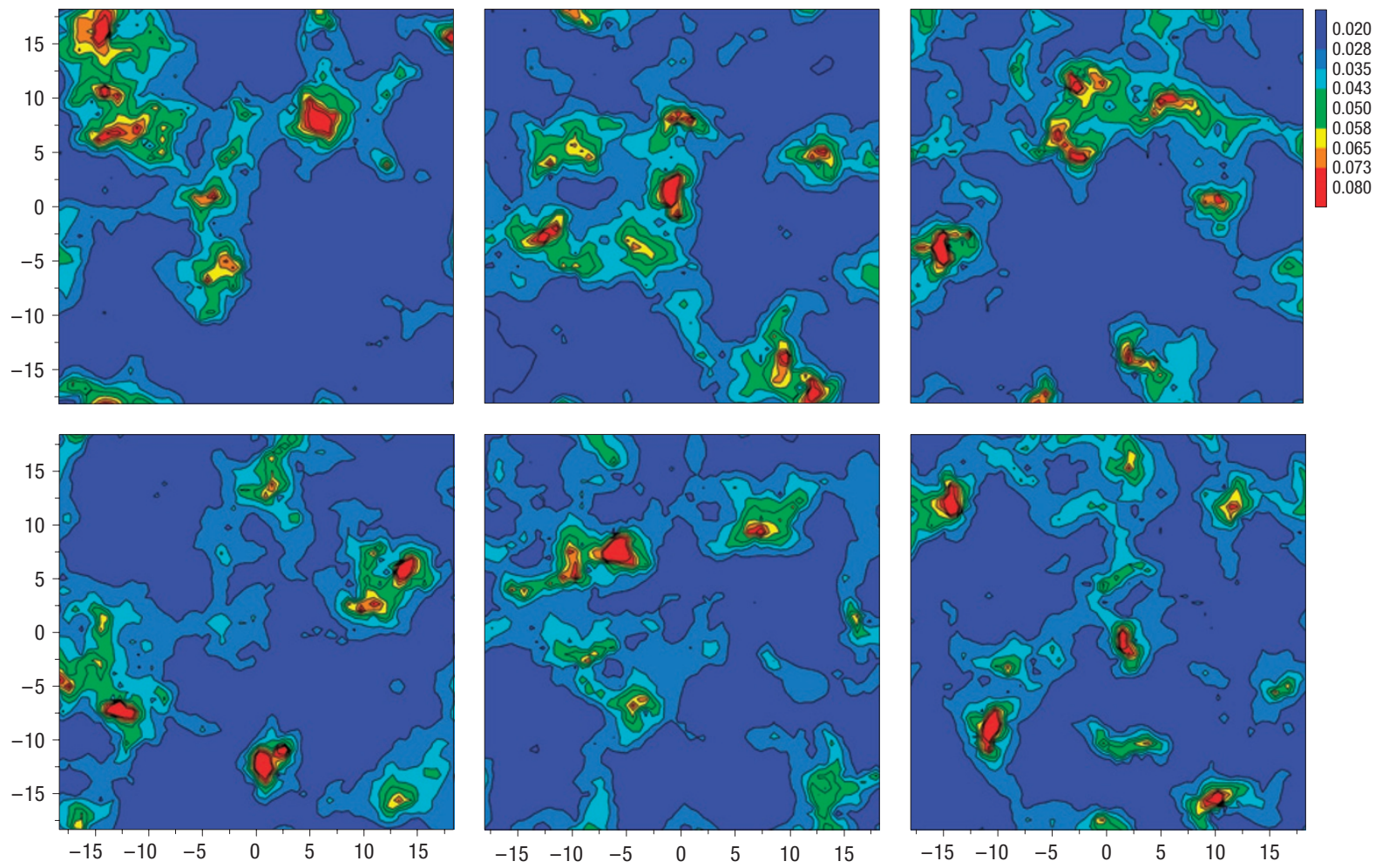

Figure 2 The spatial distribution of the low-frequency normal modes. Contour plots of the participation fraction summed over the 30 lowest-frequency modes for the quenched initial configurations of the same six configurations as used in Fig. 1. 

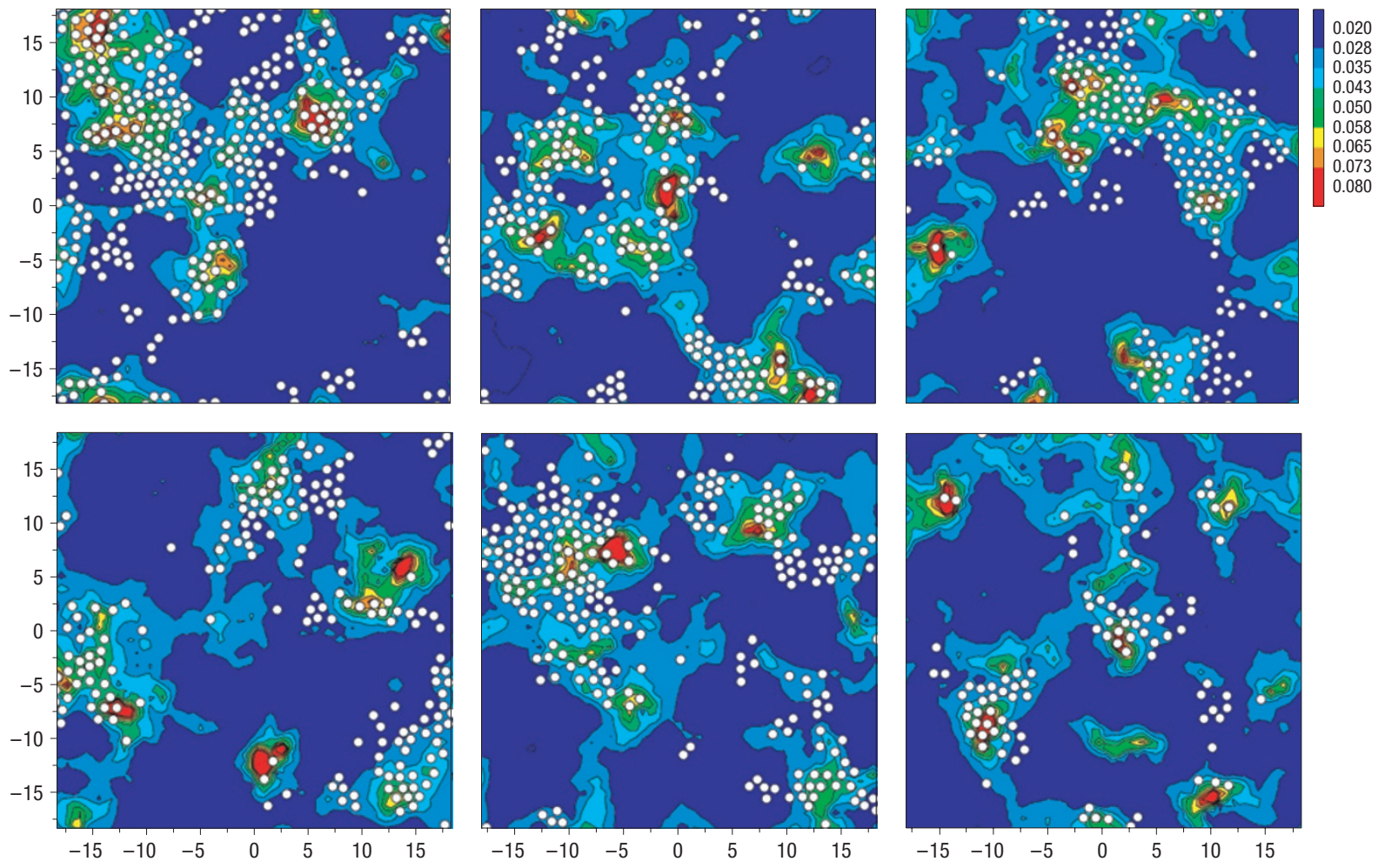

Figure 3 A comparison of the spatial distribution of IR and low-frequency normal modes. Contour plots of the low-frequency mode participation (as in Fig. 2), overlaid with the location of particles (white circles) whose iso-configurational probability of losing four initial nearest neighbours within $200 \tau$ is greater than or equal to 0.01 .

Two points are worth emphasizing. The mode participation fractions, whose spatial distributions are mapped in Fig. 2, are properties of the static initial configurations. Our demonstration of a strong correlation between the mode maps and the irreversible reorganization maps constitutes a significant success in understanding how structure determines relaxation in an amorphous material. Indeed, as Fig. 2 illustrates, we may provide semiquantitative prediction of IR domains as they emerge at relatively long times from the initial configuration alone. The second point is that, because we have used quenched modes, we have used only information about the bottom of the local potential minima. While it is quite possible that the timescale required for a reorganization event will depend on the energy barriers associated with the transition, our results indicate that the spatial structure of such events is largely determined by the distribution of soft quasilocalized modes in the initial configuration.

Given our conclusion that relaxation originates with soft quasilocalized modes, it follows that our capacity to predict the subsequent spatial distribution of the IR depends on how persistent the mode distribution is in a configuration. After all, should the mode maps evolve rapidly then the structural information in a given configuration would quickly become irrelevant. The fact that we observe strong spatial correlations between the initial modes and relaxation some $200 \tau$ later indicates that the spatial structure of the modes does generally persist over such times. This is remarkable given that small variations in the quenched modes, indicative of a change in the local minimum (or inherent structure), occur over $\sim 1 \tau$ intervals. Details of these rapid changes are provided in the Supplementary Information. We conclude that the spatial structure of the quenched soft modes can often persist over many changes in the inherent structure. Preliminary results indicate that this persistence is also found in three-dimensional mixtures (including temperatures below the empirical mode-coupling temperature) (see the Supplementary Information).

We do, however, see configurations where the mode structure is not so stable. An example of this is shown in Fig. 4. In Fig. 4a,b we compare the mode participation map for the initial configuration with the map of the maximum participation fraction observed per particle over five $10 \tau$ runs starting from the configuration in Fig. $4 \mathrm{a}$. The difference in spatial structure between these maps is a measure of the degree of variability of the mode structure. In Fig. 4c,d we overlay the particles showing IR within $200 \tau$ (as defined in Fig. 3) over the maps of Fig. 4a,b, respectively. While the mode structure of the initial configuration does not provide a quantitative predictor of the spatial distribution of IR (Fig. 4c), the cumulative mode structure sampled over the multiple short runs does (Fig. 4d). This result demonstrates that, even when the soft-mode structure is not stable, the IR still originates with these soft modes, but now this IR is not well predicted by any single configuration. It seems that configurations such as that analysed in Fig. 4 represent those caught in transit between configurations with more stable mode structure.

In this paper we have presented two important results relating to the slow relaxation in a model supercooled liquid. The first is that the irreversible reorganization originates at the sites of the low-frequency quasilocalized quenched modes. The second is that these modes typically persist for timescales significantly longer than the lifetime of a given inherent structure. These results show that the spatial location and extent of IR regions at relatively long times may be reasonably predicted by a simple, static property of the static initial condition. A number of previous reports have linked localized dynamics with the presence of soft modes ${ }^{16-20}$ during or immediately before the appearance of the motion in 

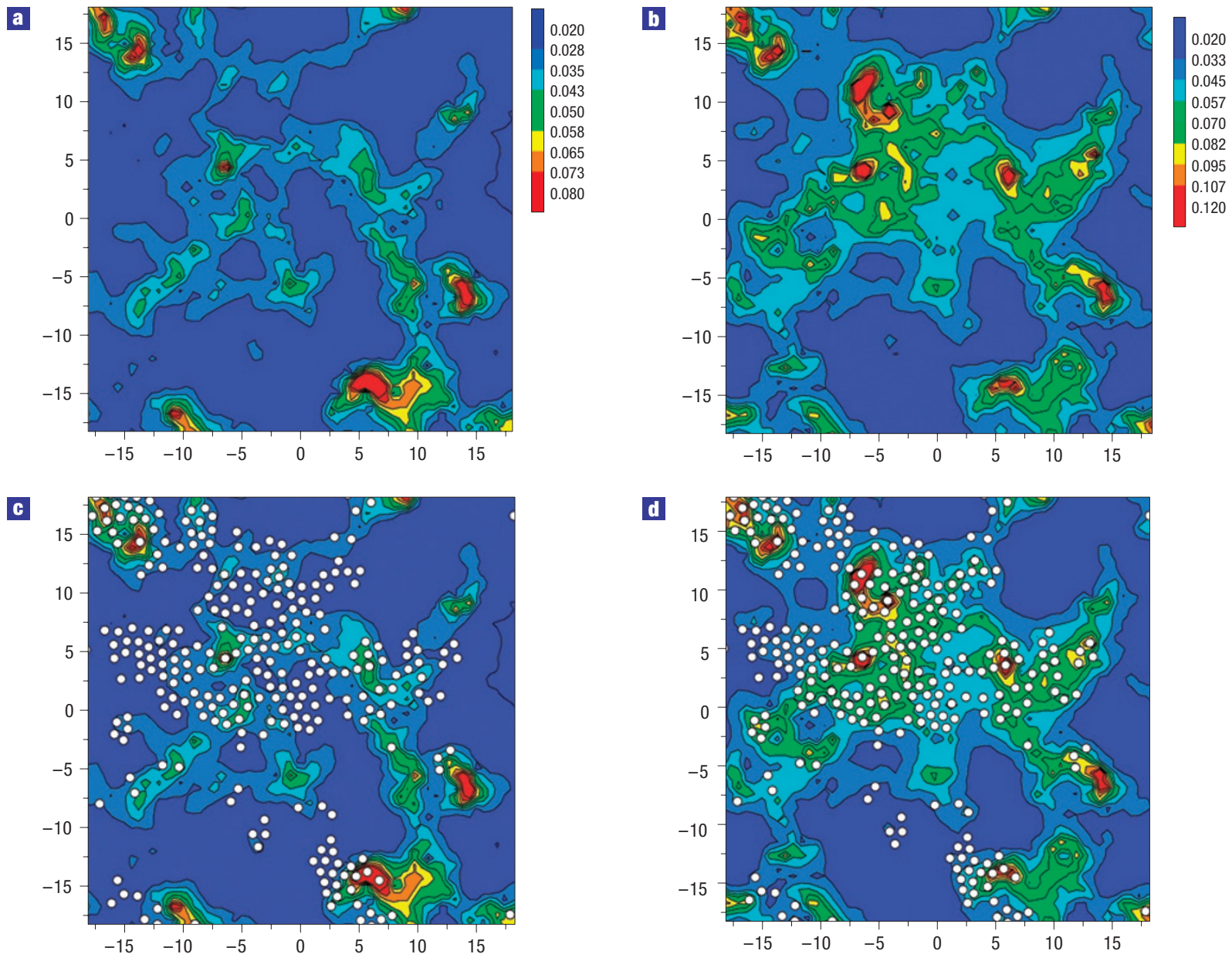

Figure 4 The consequences of rapid variations in normal mode structure. a, Contour plot of the participation fraction summed over the 30 lowest-frequency modes for a quenched configuration. $\mathbf{b}$, Contour plot of the maximum value of the participation fraction observed over five $10 \tau$ runs starting from the configuration in a. c, Particles whose iso-configurational probability of losing four initial nearest neighbours within $200 \tau$ is greater than or equal to 0.01 (white circles) overlaid on the participation fraction map for the initial configuration. $\mathbf{d}$, As in c except that the overlay is over the map of the maximum participation fraction shown in $\mathbf{b}$.

question. In contrast, we have demonstrated that soft localized modes are typically present in configurations well in advance of the appearance of the IR associated with them. The results of this paper suggest that the quasilocalized modes can provide a unification of many of the major themes in current research on the glass transition. Can the growth in the four-point susceptibility $\chi_{4}$ near the glass transition ${ }^{6,21}$ and the jamming transition in granular material $^{22}$, along with the growth in the related kinetic correlation length, be directly connected to an equilibrium correlation function of the soft-mode distribution? A recent paper $^{23}$ has examined the localization of vibrational modes in granular material. Does the persistence of the mode structure in real space reflect the transient confinement of the system trajectory within a 'metabasin' in configuration space ${ }^{24}$ ? How both the spatial character of the quasilocalized normal modes and their correlation with subsequent relaxation varies with temperature remains the most fundamental question raised by this paper and is the subject of ongoing research. We have established that these quasilocalized modes represent the strongest causal link yet established between structure and dynamic heterogeneity and, hence, an exciting route forward to establish how molecular properties influence relaxation in the supercooled liquid.

\section{METHODS}

For a glass-forming liquid, we use a two-dimensional equimolar binary mixture of particles interacting via purely repulsive potentials of the form

$$
u_{a b}(r)=\varepsilon\left[\frac{\sigma_{a b}}{r}\right]^{12},
$$

where $\sigma_{12}=1.2 \times \sigma_{11}$ and $\sigma_{22}=1.4 \times \sigma_{11}$. All units quoted will be reduced so that $\sigma_{11}=\varepsilon=m=1.0$, where $m$ is the mass of both types of particle. Specifically, the reduced units of time are given by $\tau=\sigma_{11}(m / \varepsilon)^{1 / 2}$. The average collision time at $T=0.4$ in the binary mixture is $0.1 \tau$. The reduced units of temperature are $k_{\mathrm{B}} / \varepsilon$. A total of $N=1,024$ particles were enclosed in a square box with periodic boundary conditions. Molecular dynamics simulations were carried out in the NPT ensemble using a Nosé-PoincaréAndersen algorithm developed by Sturgeon and Laird ${ }^{25}$. The structural or 'alpha' relaxation time $\tau_{\alpha}$ is defined as the time required for the self intermediate scattering function $F_{\mathrm{s}}(q, t)$,

$$
F_{\mathrm{s}}(q, t)=\frac{1}{N}\left\langle\sum_{j=1}^{N} \exp \left\{i \mathbf{q} \cdot\left[\mathbf{r}_{j}(0)-\mathbf{r}_{j}(t)\right]\right\}\right\rangle,
$$

to decay to a value of $1 / e$. The magnitude of the wavevector $q$ is set equal to the value at the first Bragg peak. 
For the normal-mode analysis, the inherent structure of each configuration was found using the conjugate-gradient method. The dynamical matrix of the inherent structure was then defined, $\mathbf{D}=\left(\partial^{2} \Phi(r) / \partial r_{i}^{k} \partial r_{j}^{k}\right)$, where $r_{i}^{k}$ is the $k^{\text {th }}$ component of the position $\mathbf{r}_{i}$ of particle $i, \Phi(r)=\sum_{i=0}^{N} \sum_{j=1, j \neq i}^{N} u_{a b}\left(r_{i j}\right)$, $u_{a b}(r)$ is the intermolecular potential from equation (1) and $r_{i j}=\left|\mathbf{r}_{i}-\mathbf{r}_{j}\right|$. The dynamical matrix was diagonalized using the template numerical toolkit (http://math.nist.gov/tnt/index.html).

To visualize the spatial distribution of the propensity, it is useful to remove the additional complexity of the configuration and use contour plots. As the data points are located at irregularly spaced particle coordinates, it is necessary to interpolate between them. We have used the modified version of Shepard's method as implemented in the NAG libraries.

Received 16 January 2008; accepted 13 June 2008; published 20 July 2008.

\section{References}

1. Debenedetti, P. G. \& Stillinger, F. H. Supercooled liquids and the glass transition. Nature 410, $259-267$ (2001)

2. Angell, C. A. Formation of liquids from glasses and biopolymers. Science 267, 1924-1935 (1995).

3. Swallen, S. F. et al. Organic glasses with exceptional thermodynamic and kinetic stability. Science 315 , $353-356$ (2007).

4. Sastry, S., Debenedetti, P.G. \& Stillinger, F. H. Signatures of distinct dynamical regimes in the energy landscape of a glass-forming liquid. Nature 393, 554-557 (1998).

5. Weeks, E. R., Crocker, J. C., Levitt, A. C., Schofield, A. \& Weitz, D. A. Three-dimensional direct imaging of structural relaxation near the colloidal glass transition. Science 287, 627-631 (2000).

6. Berthier, L. et al. Direct experimental evidence of a growing length scale accompanying the glass transition. Science 310, 1797-1800 (2005).

7. Ediger, M. D. Spatially heterogeneous dynamics in supercooled liquids. Ann. Rev. Phys. Chem. 51, 99-128 (2000).

8. Kob, W., Donati, C., Plimpton, S. J., Poole, P. H. \& Glotzer, S. C. Dynamical heterogeneities in a supercooled Lennard-Jones liquid. Phys. Rev. Lett. 79, 2827-2830 (1997).

9. Hurley, M. M. \& Harrowell, P. Non-Gaussian behavior and the dynamical complexity of particle motion in a dense two-dimensional liquid. J. Chem. Phys. 105, 10521-10526 (1996).

10. Widmer-Cooper, A. \& Harrowell, P. Predicting the long-time dynamic heterogeneity in a supercooled liquid on the basis of short-time heterogeneities. Phys. Rev. Lett. 96, 185701 (2006).

11. Widmer-Cooper, A. \& Harrowell, P. Free volume cannot explain the spatial heterogeneity of Debye-Waller factors in a glass-forming binary alloy. J. Non-Cryst. Solids 352, 5098-5102 (2006).

12. Widmer-Cooper, A., Harrowell, P. \& Fynewever, H. How reproducible are dynamic heterogeneities in a supercooled liquid? Phys. Rev. Lett. 93,135701 (2004).

13. Rabani, E., Gezelter, J. D. \& Berne, B. J. Calculating the hopping rate for self-diffusion on rough potential energy surfaces: Cage correlations. J. Chem. Phys. 107, 6867-6876 (1997).
14. Volmayer-Lee, K. Single particle jumps in a binary Lennard-Jones system below the glass transition. J. Chem. Phys. 121, 4781-4794 (2004).

15. Perera, D. \& Harrowell, P. Relaxation dynamics and their spatial distribution in a two-dimensional glass-forming mixture. J. Chem. Phys. 111, 5441-5454 (1999).

16. Mazzacurati, V., Ruocco, G. \& Sampoli, M. Low-frequency atomic motion in a model glass. Europhys. Lett. 34, 681-686 (1996)

17. Schober, H. R. \& Ruocco, G. Size effects and quasilocalized vibrations. Phil. Mag. 84, 1361-1372 (2004).

18. Schober, H. R. \& Laird, B. B. Localized low-frequency vibrational modes in glasses. Phys. Rev. B 44, 6746-6754 (1991)

19. Brito, C. \& Wyart, M. Heterogeneous dynamics, marginal stability and soft modes in hard sphere glasses. J. Stat. Mech. 76, L08003 (2007).

20. Coslovich, C. \& Pastore, G. Are there localized saddles behind the heterogeneous dynamics of supercooled liquids? Europhys. Lett. 75, 784-790 (2006).

21. Berthier, L. et al. Spontaneous and induced dynamic fluctuations in glass formers. I. General results and dependence on ensemble and dynamics. J. Chem. Phys. 126, 184503 (2007).

22. Lechenault, F., Dauchot, O., Biroli, G. \& Bouchaud, J.-P. Critical scaling and heterogeneous superdiffusion across the jamming/rigidity transition of a granular glass. Preprint at $<$ http://arxiv.org/abs/0706.1531> (2007).

23. Zeravcic, Z., van Saarloos, W. \& Nelson, D. R. Localization behavior of vibrational modes in granular packings. Preprint at $<$ http://arxiv.org/abs/0802.3440> (2008).

24. Doliwa, B. \& Heuer, A. What does the potential energy landscape tell us about the dynamics of supercooled liquids and glasses? Phys. Rev. Lett. 91, 235501 (2003).

25. Sturgeon, J. B. \& Laird, B. B. Symplectic algorithm for constant-pressure molecular dynamics using a Nosé-Poincaré thermostat. J. Chem. Phys. 112, 3474-3482 (2000).

Supplementary Information accompanies this paper on www.nature.com/naturephysics.

\section{Acknowledgements}

We would like to thank L. Berthier, G. Biroli, J. P. Bouchaud, A. Heuer, and C. O'Hern for discussions. H.P. and D.R.R. would like to thank P. Verrocchio for providing the equilibrated three-dimensional configurations and NSF for financial support. A.W.-C. and P.H. acknowledge the support of the Australian Research Council. H.P. was partially supported by the MRSEC program of the National Science Foundation under grant No DMR-0213574.

\section{Author contributions}

P.H. and D.R.R. conceived this study and wrote the paper. A.W.-C. carried out the MD simulation, analysed the motions and devised the contour plots. H.P. carried out the normal-mode analysis and graphical representation of the eigenfunctions.

\section{Author information}

Reprints and permission information is available online at http://npg.nature.com/reprintsandpermissions. Correspondence and requests for materials should be addressed to P.H. or D.R.R. 